\title{
Neurokinin-1 receptor antagonist aprepitant and radiotherapy, a successful combination therapy in a patient with lung cancer: A case report
}

\author{
MIGUEL MUÑOZ ${ }^{1}$, JUAN CARLOS CRESPO ${ }^{2}$, JOSÉ PEDRO CRESPO ${ }^{3}$ and RAFAEL COVEÑAS ${ }^{4}$ \\ ${ }^{1}$ Research Laboratory on Neuropeptides (IBIS), Virgen del Rocío University Hospital, 41013 Sevilla; \\ ${ }^{2}$ Department of Pharmacology, Pediatric and Radiology, University of Sevilla, 41009 Sevilla; \\ ${ }^{3}$ SEVISTON Laboratory Pathology, 41001 Sevilla; ${ }^{4}$ Institute of Neurosciences of Castilla y León (INCYL), \\ Laboratory of Neuroanatomy of the Peptidergic Systems (Lab. 14), University of Salamanca, 37007 Salamanca, Spain
}

Received October 29, 2018; Accepted April 17, 2019

DOI: $10.3892 / \mathrm{mco} .2019 .1857$

\begin{abstract}
Lung cancer (LC) is the leading cause of cancer death and is responsible for more than one-quarter (27\%) of all cancer deaths. Non-small-cell LC (NSCLC) is the main histological subtype of LC and is the leading cause of mortalities associated with cancer. This case report focused on a patient showing a history of chronic obstructive pulmonary disease (COPD) with a NSCLC squamous cell carcinoma (single mass with a diameter of $8 \times 7 \mathrm{~cm}$, without metastasis). Due to the COPD, neither a surgical treatment (pneumonectomy) nor chemotherapy was possible. After 15 days following the diagnosis, the patient was treated for 45 days with radiotherapy and the neurokinin-1 receptor, aprepitant (compassionate use, $1,140 \mathrm{mg} /$ day). Radiotherapy was administered to the right lung and mediastinum, reaching $50.4 \mathrm{~Gy}$ and then overprinted to 65 Gy. In each successive control, the tumor volume decreased and, after 6 months of treatment, the chest computed tomography scan showed that the tumor mass had disappeared. No side-effects were observed during the combination therapy; in contrast, the patient was in very good general health with a weight gain and showing no biochemical analytical alteration. This case report suggests a witness episode to the antitumor effect of aprepitant/radiotherapy against NSCLC. Urgent development is required for clinical trials to test the combination treatment (radiotherapy and aprepitant) as an antitumor therapy for patients with LC.
\end{abstract}

Correspondence to: Dr Miguel Muñoz, Hospital Infantil Universitario Virgen del Rocío, Unidad de Cuidados Intensivos Pediátricos, Av. Manuel Siurot s/n, 41013 Sevilla, Spain

E-mail:mmunoz@cica.es

Key words: neurokinin-1 receptor, substance P, non-small cell lung cancer, non-small cell lungh cancer, antitumor, Emend, PET, chronic obstructive pulmonary disease

\section{Introduction}

Lung cancer (LC) is the primary cause of cancer-related deaths in the western world, LC being responsible for more than one-quarter (27\%) of all cancer deaths (1). Non-small-cell LC (NSCLC) is the main histological subtype of LC and the leading cause of cancer death (2). The last few decades have seen no significant progress in extending the survival of LC patients despite the multiple clinical trials using cytotoxic chemotherapeutic agents, anti-growth factor-signaling agents and/or radiotherapy (3). Because cytostatic drugs are not specific against cancer cells, they show a low safety profile and cause severe side-effects. Cancer research must now focus on strategies showing similar or higher antitumor effects, but with far fewer side-effects. Thus, safer and more specific/effective drugs against LC must be investigated along with the seeking and identification of novel molecular targets that destroy cancer cells.

One of these novel molecular targets could be the substance $\mathrm{P}(\mathrm{SP})$ /neurokinin-1 receptor (NK-1R) system (4-6). This system is known to play an important role in cancer (e.g., neuroblastoma, retinoblastoma, melanoma, hepatoblastoma, LC, etc.) (4-6). It has been reported that SP and NK-1R antagonists [aprepitant $\left(\right.$ Emend $\left.^{\circledR}\right)$, L-733,060, L-732,138] promote the proliferation and inhibition of human NSCLC and small-cell LC (SCLC) cells $(5,6)$, respectively. Both human LC cells express isoforms of the NK-1R and mRNA for the NK-1R; they overexpress the tachykinin 1 gene; NK-1R is also known to be involved in their viability $(5,6)$. In a concentration-dependent manner, NK-1R antagonists inhibited the growth of NSCLC and SCLC cell lines, the action being mediated by the NK-1R (inducing LC cells to die by apoptosis) (5,6). All together, the data suggest that the NK-1R is a promising target in the treatment of cancer (e.g., LC) and that NK-1R antagonists behave as potential antitumor drugs. The NK-1R antagonist aprepitant was administered here because it is a drug currently administered in clinical practice for the treatment of nausea and vomiting induced by chemotherapy and because it is safe and well-tolerated (7-9). Thus, aprepitant is a good candidate to be tested in patients in which neither a surgical treatment (pneumonectomy) nor chemotherapy is possible. This is the case of the patient studied here. 
A

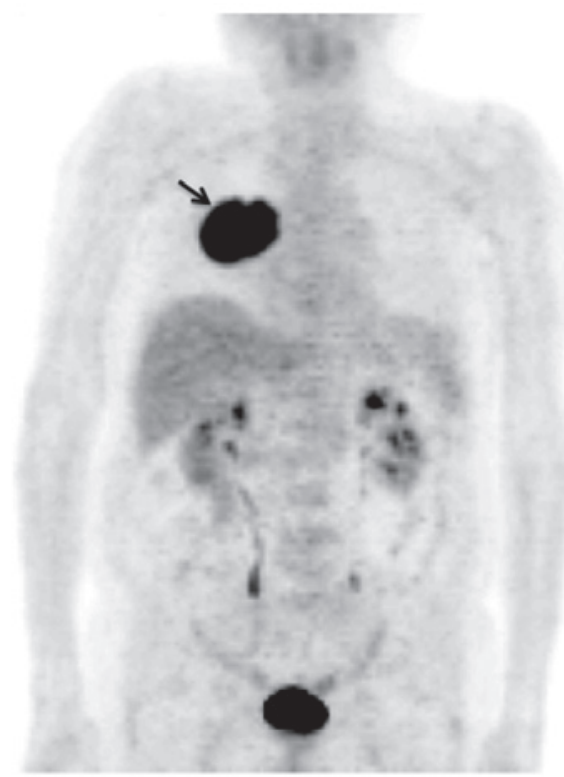

B

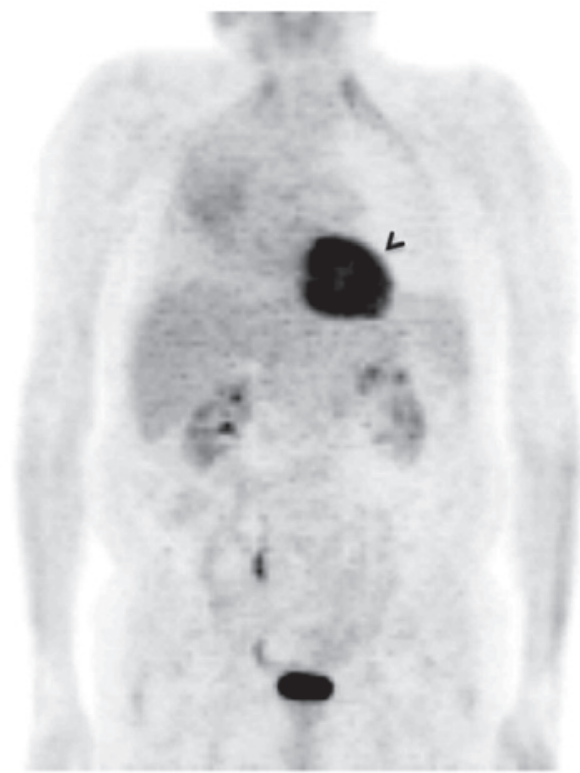

Figure 1. Whole body PET. (A) This image was taken when the patient was diagnosed with NSCLC. An intense FDG uptake was visualized in the lower portion of the right upper lobe $(7 \times 8 \mathrm{~cm}$; arrow). Note that, in the heart, the FDG uptake was very low. (B) Six months after the treatment was started. The great lung pathological focus, observed six months before, is not evident. The intense FDG uptake observed in Fig. 1A, in the right lung, disappeared and only a diffuse uptake of FDG was observed. By contrast, the heart (arrowhead) showed a higher FDG uptake (physiological uptake) than that showed in the right lung, but similar to the uptake of the tumor mass when the patient was diagnosed. In both images, the rest of the FDG uptake was located in the excretory system.

\section{Case report}

Patient and treatment. Male (76 years; height: $187 \mathrm{~cm}$; body weight: $82 \mathrm{~kg}$ ) diagnosed with squamous cell carcinoma in the right main bronchus up to 2 -cm from the carina. About $8 \times 7 \mathrm{~cm}$ in diameter and, according to the PET, forming a single mass without metastasis (Fig. 1A). No mediastinal lymphadenopathy. Performance status (PS): Moderate. Respiratory function tests showed that the patient was, at the limit, for pneumonectomy. Histopathology: Moderate differentiated squamous cell carcinoma. CEA (carcinoembryonic antigen): Normal. Diagnostic: NSCLC. Staging of the patient was T4N3M0. Surgical treatment (pneumonectomy) was not possible due to the proximity of the tumor mass to the right main bronchus and because the patient had chronic obstructive pulmonary disease (COPD) with limited respiratory functions (the patient needed non-invasive mechanical home ventilation). Thus, chemotherapy was not possible either. Treatment: Combination therapy with radiotherapy and the NK-1R antagonist aprepitant (compassionate use). The treatment was authorized by the Spanish Medication Agency and the Spanish Ministry of Health. Regime of the treatment was (45 days): 1) Radiotherapy on the right lung and mediastinum (reaching up to $50.4 \mathrm{~Gy}$ (25 sessions) and then overprinted to $65 \mathrm{~Gy}$ (8 additional sessions) and 2) administration of aprepitant (1,140 mg/day). Thus, fifteen days after diagnosis, radiotherapy and aprepitant were administered simultaneously. According to the results obtained during in vitro pre-clinical studies, the amount of the NK-1R antagonist used in these studies $(20 \mu \mathrm{M}$ approximately) $(10,11)$, was extrapolated to the patient studied here.

Clinical course. Three weeks after treatment was started (Table I), no side-effects from aprepitant were observed. The evolution was, from a clinical point of view, good. Karnosfky: 90. Good appetite. The patient increased in body weight from 82 to $87 \mathrm{~kg}$. Physical examination: Good PS, coloration and hydration of skin/mucous membranes. Dry radiodermatitis: Field grade I. In both lungs: No dyspnea or vesicular murmur. No lymphadenopathies. In abdomen, visceromegaly was not observed. Chest CT showed a decrease of the lung mass $(5.9 \times 3.8 \mathrm{~cm}$; initially $7 \times 8 \mathrm{~cm}$ in diameter). The nodule of the posterior segment of the right upper lobe was $4 \mathrm{~mm}$, initially $7 \mathrm{~mm}$. No other injuries were observed. The hemogram revealed a discrete anemia $(\mathrm{Hb}$ : $10.6 \mathrm{~g} / \mathrm{dl})$. Leucopenia grade I (3,400 leukocytes). Platelet level: Normal. The erythrocyte sedimentation rate (ESR) was reduced from 95 to $27 \mathrm{~mm}$. Biochemical tests: No alteration.

Five weeks after the treatment started (Table I), the physical examination showed a very good PS. Body weight: $90 \mathrm{~kg}$. No dyspnea or expectoration. Good appetite. Exploration: Sibilants in the entire right lung. No peripheral adenopathy. Abdomen did not show visceromegaly. ESR: $25 \mathrm{~mm}$. Hb: 10.6 g/dl. Leukocytes: 5,200. Glycaemia, transaminases, GGT and alkaline phosphatase: Normal levels. Chest CT scan: Right hemitorax, the pulmonary effusion increased as well as density at the level of the lower lobe, showing undefined borders and a tendency to coalesce. The right supraparahilar mass did not show variations and loss of volume associated with the right lobe was not found.

Six months after the treatment started (Table I; Fig. 1B), the patient showed a very good PS. Weight: $90.4 \mathrm{~kg}$. No dyspnea or spitting. Good appetite. Exploration: Wheezing or sibilants in the entire right lung. Left lung: Normal pulmonary auscultation. No peripheral lymphadenopathy or visceromegalies. ESR: 25 mm. Hb: $13.5 \mathrm{~g} / \mathrm{dl}$. Leukocytes: 5,200. Chest CT scan: Right upper lobe collapse. Pleural effusion of medium-low quantity. No tumor mass was observed. Hilum and mediastinal adenopathies were not observed. In the right lung, PET showed 


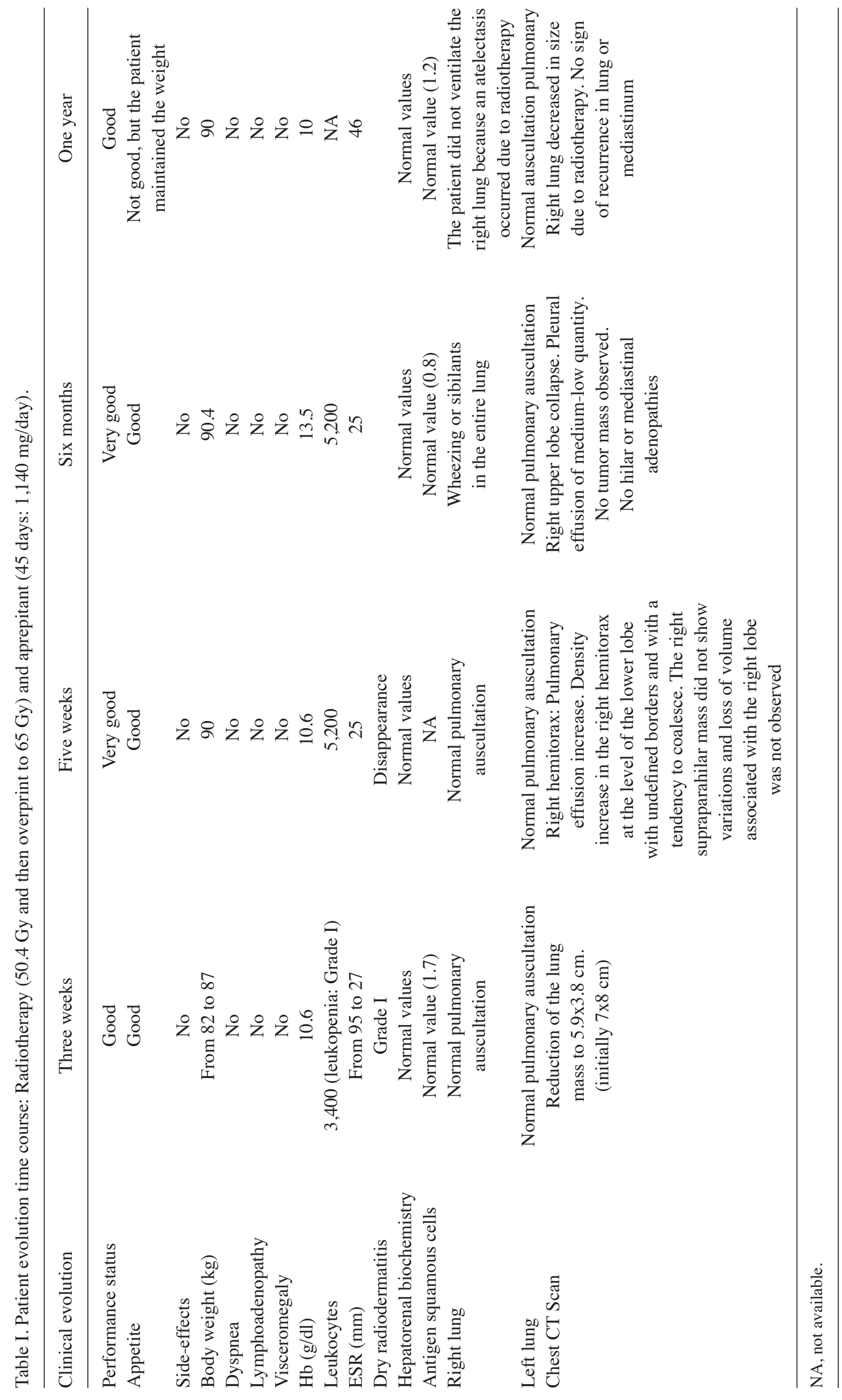


a great decrease of the uptake which was seen to be diffused (Fig. 1B) compared to that observed when the patient was first diagnosed with LC (Fig. 1A). In summary, excellent response to treatment. No current neoplastic activity was observed.

One year later (Table I). PS: Good (e.g., the patient walks frequently). No dyspnea. Dry cough without changes. Appetite: Not good, but the patient has maintained the weight gained. Physical examination showed no peripheral adenopathy. Left lung: Normal pulmonary auscultation. The patient did not ventilate the right lung, because an atelectasis appeared due to radiotherapy. Abdomen did not show visceromegaly. $\mathrm{Hb}$ : 10 g/dl. ESR: 46 mm. Normal hepatorenal biochemistry. Antigen squamous cells: 1.20. Chest CT scan showed that the right lung decreased in size, due to radiotherapy, and no sign of recurrence in the lung/mediastinum was observed.

Two years later. Chest CT scan showed that the right lung decreased in size due to radiotherapy. Compensatory pleural effusion, without masses or ganglia, was observed. Lung or mediastinum: No sign of recurrence was observed. Abdomen CT scan: Normal. In summary, no sign of LC.

Three years later. Similar results to those observed at two years. Due to causes not related to NSCLC, the patient died.

\section{Discussion}

The current treatment for NSCLC is surgical intervention, chemotherapy and radiotherapy. However, the patient studied here could not be treated surgically because of having chronic obstructive pulmonary disease (COPD) and, due to the poor prognosis of the disease, chemotherapy was not administered. Thus, the impossibility of carrying out either surgical treatment or the administration of chemotherapy, darkened the prognosis.

Attempting to improve his life expectancy and according to the results obtained in pre-clinical studies $(10,11)$, the NK-1R antagonist aprepitant was administered (compassionate use). The amount of the NK-1R antagonist used in these pre-clinical studies ( $20 \mu \mathrm{M}$ approximately) was extrapolated $(1,140 \mathrm{mg} /$ day $)$ to the patient studied here. Moreover, in other pre-clinical study, it was fully demonstrated that aprepitant, in a concentration-dependent manner, promoted the apoptosis of NSCLC cells (tumor cells die by apoptosis); that these cells overexpress the NK-1R and that the NK-1R is involved in its viability (5). In a clinical trial to treat moderate to severe depression, it was found that a dose of $300 \mathrm{mg} /$ day of aprepitant was safe, well-tolerated and showed side-effects similar to the placebo (7). It is also known that administration of aprepitant ( $375 \mathrm{mg} / \mathrm{day})$ to HIV patients for 2 weeks was safe, well-tolerated and decreased the number of $\mathrm{CD}^{+}$ PD-1-positive cells along with the plasma level of SP (8). It is known that LC cells express PD-L1 (12). Additionally, it has been reported that a patient, unmanageable to all standard antiemetic therapies, suffering from breast cancer brain metastasis, received aprepitant $(80 \mathrm{mg} / \mathrm{day}$; initially for seven months) followed by an increase to $120 \mathrm{mg}$ of the drug every third day. In this patient, clinical conditions were improved, the level of the CA153 tumor marker decreased (from 187 to 122), no side-effects and good control of nausea/vomiting were observed (13).

It is known that the PET technique, using labeled fluoro deoxy glucose (FDG), is based on the Warburg effect. This effect occurs in most cancer cells which pre-dominantly produce energy by means of a high rate of glycolysis followed by lactic acid fermentation (14). Growing cancer cells show glycolytic rates up to 200 times higher than those of their normal tissues of origin and this occurs even if oxygen is plentiful (14). SP, in a concentration-dependent manner, promotes glycogen breakdown and favors the Warburg effect and then the glucose obtained would be used by cancer cells to increase their metabolism (4). NK-1R antagonists inhibit this effect which means that the action is mediated by the NK-1R and that these antagonists could produce death of cancer cells by starvation $(4,15)$. NSCLC express SP and NK-1Rs and hence aprepitant could block the glycogen breakdown in these cells, counteracting the Warburg effect (4). Currently, the Warburg effect is exclusively applied in clinical practice for diagnosis (PET); however, it is also possible to take advantage of this mechanism (as an antitumor strategy) by using NK-1R antagonists (e.g., aprepitant) (4).

It is also known that radiotherapy elicits neurogenic inflammation (vasodilatation, increase the permeability of blood vessels, extravasation of plasma proteins) and that this inflammation is mediated by the NK-1R (4). Thus, it seems that aprepitant could exert a dual effect: Antitumor activity (against the NSCLC cells overexpressing the NK-1R) (4-6) and to decrease the side-effects promoted by radiotherapy (16). In the patient studied here, aprepitant could have exerted this dual effect. This idea is in agreement with the results found here, since the patient did not have nausea/vomiting and showed good general health condition (it has also been reported that aprepitant exerts an antidepressant action) (7). Moreover, despite radiotherapy, the anemia did not get worse and only presented leucopenia grade I (3,400 leukocytes). Two weeks after the treatment was started, the number of leukocytes returned to normal values. In biochemical tests, no alteration was observed due to the treatment with aprepitant. This means that aprepitant (1,140 mg/day for 45 days) was safe and well-tolerated and that the combination therapy of radiotherapy and aprepitant (this NK-1R antagonist promotes the apoptosis in cancer cells overexpressing the NK-1R) (4-6,9-11) could be a good strategy against LC.

Finally, the USA National Comprehensive Cancer Network (NCCN) recommends that the best management for cancer patients (having a median overall survival of approximately 1 year) and in which current therapeutic strategies failed, is to be included in clinical trials testing new antitumor strategies (17). In this case, the NCCN encourages the participation of these patients in clinical trials. Thus, the case studied here opens the door to develop in patients with LC a clinical trial to test the use of aprepitant alone or in combination therapy with radiotherapy.

\section{Acknowledgements}

The authors would like to thank Ms Diane Haun (University of Utah, USA) for reviewing the English of the manuscript.

\section{Funding}

No funding was received. 


\section{Availability of data and materials}

The datasets used and/or analyzed during the present study are available from the corresponding author on reasonable request.

\section{Authors' contributions}

MM conceived the study. MM and JCC performed the research. JCC and JPC were responsible for clinical diagnosis. MM and RC designed and supervised the study, collected, analyzed and interpreted the data, and drafted the manuscript. All the authors read and approved the final manuscript.

\section{Ethics approval and consent to participate}

The compassionate use of aprepitant was authorized by the Spanish Medication Agency and the Spanish Ministry of Health. All procedures performed in this study were followed in accordance with the Helsinki Declaration. Written informed consent was obtained from the patient.

\section{Patient consent for publication}

Written informed consent was obtained from the patient.

\section{Competing interests}

USPTO Application no. 20090012086 'Use of non-peptidic NK-1 receptor antagonists for the production of apoptosis in tumor cells' (Miguel Muñoz).

\section{References}

1. Siegel RL, Miller KD and Jemal A: Cancer statistics, 2016. CA Cancer J Clin 68: 7-30, 2016.

2. Subramanian J, Regenbogen T, Nagaraj G,Lane A,Devarakonda S, Zhou $\mathrm{G}$ and Govindan R: Review of ongoing clinical trials in non-small-cell lung cancer: A status report for 2012 from the clinicaltrials.gov web site. J Thorac Oncol 8: 860-865, 2013.
3. Hodkinson PH, MacKinnon A and Sethi T: Targeting growth factors in lung cancer. Chest 133: 1209-1216, 2008.

4. Muñoz M and Coveñas R: Involvement of substance $P$ and the NK-1 receptor in cancer progression. Peptides 48: 1-9, 2013.

5. Muñoz M, González-Ortega A, Rosso M, Robles-Frías MJ, Carranza A, Salinas-Martín MV and Coveñas R: The substance P/neurokinin-1 receptor system in lung cancer: Focus on the antitumor action of neurokinin-1 receptor antagonists. Peptides 38: 318-325, 2012.

6. Muñoz M, Rosso M and Coveñas R: Neurokinin-1 receptor antagonists in lung cancer therapy. Lett Drug Des Discov 14: 1465-1476, 2017.

7. Kramer MS, Cutler N, Feighner J, Shrivastava R, Carman J, Sramek JJ, Reines SA, Liu G, Snavely D, Wyatt-Knowles E, et al: Distinct mechanism for antidepressant activity by blockade of central substance P receptors. Science 281: 1640-1645, 1998.

8. Tebas P, Tuluc F, Barrett JS, Wagner W, Kim D, Zhao H, Gonin R, Korelitz J and Douglas SD: A randomized, placebo controlled, double masked phase IB study evaluating the safety and antiviral activity of aprepitant, a neurokinin-1 receptor antagonist in HIV-1 infected adults. PLoS One 6: e24180, 2011.

9. Muñoz M and Coveñas R: Safety of neurokinin-1 receptor antagonists. Expert Opin Drugs Safety 12: 673-685, 2013.

10. Muñoz M, Pérez A, Coveñas R, Rosso M and Castro E: Antitumoural action of L-733,060 on neuroblastoma and glioma cell lines. Arch Ital Biol 142: 105-112, 2004.

11. Muñoz M, Rosso M, Pérez A, Coveñas R, Rosso R, Zamarriego C and Piruat JI: The NK1 receptor is involved in the antitumoural action of L-733,060 and in the mitogenic action of substance $\mathrm{P}$ on neuroblastoma and glioma cell lines. Neuropeptides 39: 427-432, 2005.

12. Yu H, Boyle TA, Zhou C, Rimm DL and Hirsch FR: PD-L1 Expression in lung cancer. J Thorac Oncol 11: 964-975, 2016.

13. Lee M, McCloskey M and Staples S: Prolonged use of aprepitant in metastatic breast cancer and a reduction in CA153 tumour marker levels. Int J Cancer Clin Res 3: 071, 2016.

14. Warburg O: On the origin of cancer cells. Science 123: 309-314, 1956.

15. Medrano S, Gruenstein E and Dimlich RV: Substance P receptors on human astro-cytoma cells are linked to glycogen breakdown. Neurosci Lett 167: 14-18, 1994

16. Alfieri $\mathrm{AB}$ and Cubeddu LX: Efectos de los antagonistas de los receptores NK1 y de la dexametasona sobre la inflamación neurogénica inducida por ciclofosfamida y por radiación $\mathrm{X}$, en la rata. Archivos Venezolanos de Farmacologia y Terapeutica 23: 61-66, 2004.

17. Ettinger DS, Wood DE, Aisner DL, Akerley W, Bauman J, Chirieac LR, D'Amico TA, DeCamp MM, Dilling TJ, Dobelbower M, et al: Non-small cell lung cancer, version 5.2017, NCCN clinical practice guidelines in oncology. J Natl Compr Canc Netw 15: 504-535, 2017. 